\title{
A Hybrid Agent-Based and Probabilistic Model for Fine-grained Behavioural Energy Waste Simulation
}

\author{
Fatima Abdallah, Shadi Basurra, and Mohamed Medhat Gaber \\ School of Computing and Digital Technology, Birmingham City University, Birmingham, UK \\ Email: fatima.abdallah@bcu.ac.uk, shadi.basurra@bcu.ac.uk,mohamed.gaber@bcu.ac.uk
}

\begin{abstract}
Several agent-based and probabilistic models were proposed to simulate human behaviour, which is an important cause of high energy consumption in buildings. However, some of these models ignore behavioural energy waste at occupant level, and when they model it, they are based on small case studies and produce high level energy consumption data. This paper proposes a hybrid approach that integrates agent-based and probabilistic models to simulate behavioural energy waste at occupant level. The combination of the two approaches helps produce fine-grained data, and is based on large real data samples. The developed model was validated against realistic data. The results show that employment type have an effect on the energy consumption of households, which needs further investigation to quantify the effect and test other social parameters.
\end{abstract}

\section{INTRODUCTION}

More than half of the energy consumption of buildings is caused by human behavioural energy waste (e.g. leaving appliances and lights on while not in use) [1]. Therefore, it is crucial to study human behaviour especially with the recent research in zero carbon buildings design where human behaviour is important [2].

To analyse buildings energy performance and study the effect of human behaviour, several energy simulation models have been proposed. Among these are Probabilistic Models (PM) and Agent-Based Models (ABM) that simulate energy consumption human behaviour. PM simulate the activities of occupants through probability distributions then get the resulting energy consumption of appliances [2], [3]. However, these models do not simulate occupants behavioural energy waste. They assume ideal human behaviour [4] and consider that occupants have identical behavioural characteristics, while in fact, occupants may have different consumption habits [5]. ABM approaches have been proposed to model behavioural energy waste in both commercial [6] and residential buildings [7]. In these models, occupants/energy consumers are modelled as separate computational entities that change their state and make decisions by interacting with their environment (electric appliances) and other occupants [8]. However, most of these models do not model the low level interaction between occupants and appliances thus produce high level data. Modelling this interaction at a fine-grained level is important to determine the causes of energy waste in buildings [9].

This paper proposes a hybrid approach that takes advantage of both probabilistic and agent-based models to overcome their limitations when they work separately. Thus, obtaining a model that simulates various levels of energy awareness of occupants and produces more detailed data at occupant and appliance level. This helps in understanding the impact of occupant's energy awareness levels in family settings. The results of this paper set the way for more experiments to study the effect of social parameters on the energy consumption of a family. The paper is divided into the following sections: the next section presents part of the existing probabilistic and agent-based models highlighting their limitations and showing how integrating them in one model overcomes these limitations. Section III presents the integration methodology. Section IV illustrates the resulting energy consumption of different types of households. Finally, conclusion and future work are presented in section $\mathrm{V}$.

\section{RELATED WORK}

\section{A. Probabilistic Models}

Probabilistic Models (PM) have been widely proposed to predict energy demand in residential buildings. They utilise time-use surveys to calculate the probability that an action occurs and simulate occupant activities and energy consumption at home. These models are considered as bottom-up approaches that build up the energy consumption of the building from high resolution data at activity and appliance level [3]. Bottom-up approaches make it possible to detect energy waste when having information about what the occupant is doing, what is his/her location, which appliances are turned on, etc. In addition, this level of granularity is useful to study the changes in occupant behavioural characteristics [10].

Although PM produce high resolution data which is useful when modelling energy waste, existing models only aim to reproduce realistic occupant activities and energy consumption. This is because these models follow a linear data generation process where occupancy and activity data are generated and then used to generate the resulting electricity consumption. This linear process cannot be used to model dynamic behaviour because human behaviour is non-linear and can change based on several individual and environmental attributes [8].

Existing PM assume that all occupants are the same and consume energy in an ideal way; That is, energy is consumed only when occupants are available at home or doing the activity [3], [4], [11]. However, human behaviour is more complex and is unlikely to be the same. For example, more than $50 \%$ of energy consumption in commercial buildings is consumed during unoccupied hours [1]. In addition, residential occupants can be categorised into high, medium, and low consumers [6]. Ignoring the different levels of human energy awareness have 
caused an underestimation of energy consumption compared to the real data in some existing PM. Richardson et al. [4] noticed that there is more consumption during night in the real data compared to the simulated one, and attributed this to occupants leaving lights on when they sleep. Similarly, Aerts [11] realised that the developed model failed to produce high energy consumption levels, and explained that the reason could be behavioural energy waste.

\section{B. Agent-based Models}

Besides PM, Agent-Based Models (ABM) simulate human actions in dynamic environments. In ABM, agents are defined as autonomous software components that take decisions based on their state and rules of behaviour. ABM is best used when agents behaviour is non-linear and affected by the surrounding environment, when agents location is not fixed, and when agents characteristics are heterogeneous [8].

Existing ABM approaches have been proposed for both residential and commercial buildings and for different purposes. For example, Azar and Menassa [5], [6] used ABM to study the effect of peer pressure and energy conservation workshops on the energy consumption of a commercial building. This model differentiates between occupants by varying the average yearly consumption. This factor is not only affected by how aware the occupants are, but also how long they spend in the building or what appliances they use. In addition, it does not produce high resolution data like location and activity of occupants which are important attributes when studying behavioural energy waste. In a similar way, Zhang et al. [7] represented energy-consumer agents at household level to study the experience development of households when using smart meters. Taking the household as a whole entity which has one energy awareness level makes it difficult to model occupants-appliances interaction and study the effect of occupants energy awareness on the consumption of the family.

Among existing ABM a few of them model the occupantappliance interaction and vary the energy awareness at occupant level. For instance, Carmenate et al. [9] developed an ABM that models the human-appliance-building interaction, and highlighted the effect of both building structure and occupants awareness on energy consumption of the building. The advantage of such models is that they simulate the detailed movement of occupants in the building and study the factors that affect energy consumption whether they are physical, social or others. However, the limitation of these models is that they are implemented for specific case studies which offers energy efficiency strategies specific for these environments, whereas using large samples allows for studying more varied scenarios which results in wider conclusions.

\section{Integrating Probabilistic and Agent-based models}

PM utilise large samples of data which guarantees that the produced data are realistic. They also provide high resolution data at appliance and occupant level. Therefore, PM can overcome the limitations in some of the ABM models presented above. On the other hand, ABM overcomes the linear approach in PM by enabling dynamic human behaviour modelling where occupant agents take decisions based on their personal characteristics and the external state of the environment. Furthermore, various energy awareness levels can be modelled at the occupant level in ABM which enables the study of energy awareness in a family setting. Therefore, a hybrid approach that combines $\mathrm{ABM}$ and $\mathrm{PM}$ overcomes limitations of both models when they are separated. This idea of using PM in ABM was recently proposed in Reynaud et al. [12] who propose to calibrate an ABM model with PM to gain reactivity and coordination of occupant agents. Despite the fact that the integration has not been implemented yet, the ABM they proposed do not model behavioural energy waste.

\section{The Agent-Based And Probabilistic Model INTEGRATION METHODOLOGY}

The ABM model proposed in this paper obtains realistic behaviour of occupants from Aerts PM [2], [11]. Aerts model is one of the recent models which has advantages over other models [3], [4], [10] and satisfies the requirements of modelling energy waste. The model was selected because it (1) produces more realistic occupancy and activities data, (2) enables doing more than one activity at a time, (3) includes nine activities that are linked to energy usage, and (4) distinguishes between household tasks and personal activities.

Aerts model generates realistic occupancy and activity data through probability distribution functions (PDF) extracted from Belgian Time-Use Survey and Household Budget Survey which include 6400 respondents from 3455 households. The PDFs are generated based on several parameters such as occupant ages and employment types, household types, and days of week. The model is composed of three stages: (1) occupancy modelling and (2) activity modelling which were used in the ABM to produce realistic human behaviour, and (3) electricity modelling. In order to model behavioural energy waste, modifications were made mainly on the electricity model by adding an energy awareness and location attribute for occupant agents. These attributes, along occupants activity and time of day, are used to control when occupants turn appliances and lights on or off. The ABM consists of: 'Occupants Agents', 'Appliances Agents', and the 'Environment' that the agents act in.

\section{A. The Environment and Appliances Agents}

Occupants and appliances agents act in a house environment composed of a number rooms. The house rooms affect the mobility and number of locations that the occupants can be in, and consequently their energy consumption. Therefore, the number of rooms in the house was obtained from the Eurostat income and living conditions database [13] which contains the average number of rooms per person by type of household and income group. The data was normalized and fitted to the household types included in the PM. Every household was assigned one kitchen, one living room, at least one bedroom and at least one bathroom. Dining and laundry/utility rooms were added in high income houses when necessary. The size of basic rooms was set to $20 \mathrm{~m}^{2}$ based on the average room size in Belgium [14] which was used to calculate lights consumption. In terms of the day and time, the occupant agent is aware of the day type (Weekday, Saturday, or Sunday), 
time of day (10-minute time step), and the amount of external daylight.

Electric appliances in the house are modelled as dummy agents that are controlled by occupant agents. They only respond to actions from occupants to change their state from on to off or vice versa. At every time step, each appliance records the amount of consumed energy based on its state. Before initialising the simulation, every household is assigned a number and types of appliances based on the household type and income as modelled in the PM. The type of appliance identifies the amount of energy that the appliance consumes when it is on.

Therefore, the simulation environment $\mathbf{E}$ can be define using the triplet $<\mathbf{T}, \mathbf{R}, \mathbf{A}\rangle$, where:

- $\mathbf{T}$ is a one-year simulation time defined by the triplet $<t$, $d$, daylight $t_{t d}>$ where $t \in[1-144]$ is a 10-minute time step in every day $d$, and daylight $t_{t d}$ is the amount of external daylight at every time step $t$ and day $d$.

- $\mathbf{R}$ is the set of rooms in the house. For every room $r \in$ $\mathbf{R}, r$ is defined by the triplet $<$ size, $A_{r}, O_{r}>$, where size is the size of $r, A_{r}$ is the set of appliances in $r$, and $O_{r}$ is the set of occupants that are in $r$.

- $\mathbf{A}$ is the set of appliances. For every appliance $a \in \mathbf{A}, a$ is defined by the set <inUseConsumption, $r, O_{a}, C_{t d}>$, where inUseConsumption is the amount of energy used when the device is on, $r$ is the room that the appliance is in, $O_{a}$ is the set of occupants using the appliance, and $C_{t d}$ is the consumption array of the appliance over a whole year, where every $c_{t d} \in C_{t d}$ can be either inUseConsumption or 0 based on the appliance state.

\section{B. The Occupant Agent}

Initially, occupants' ages and employment types are given as input for the model. Employment types include: full time job, part time job, unemployed, retired and school, where under 18 occupants are school children and above 65 are retired. Based on the household type (occupants' ages and employment types) the income of the household is assigned using the income PDF in the PM. Next, the appliances and rooms of the house are determined as detailed above. At every time step, the occupants change the state of the environment by changing their location and using the electric appliances.

1) Occupant Daily and Weekly Behaviour: Before simulating occupancy, work routines and occupancy patterns are assigned to each occupant. Details of these attributes can be found in Aerts et al. [11]. At every time step, the occupant either selects a new occupancy state $0 s_{t d}$ based on PDFs in the PM, or decrements the duration of an already running occupancy state. The occupant action to select new occupancy state is defined by the function OS : $\mathbf{o p}_{\mathbf{d}}, \mathbf{o s}_{(\mathbf{t}-\mathbf{1}) \mathbf{d}}, \mathbf{t} \rightarrow \mathbf{o s}_{\mathbf{t d}}$

$$
\text { op }_{\mathbf{d}}, \text { os }_{\mathbf{t d}}, \mathbf{t} \rightarrow \mathbf{d r}
$$

where, $o s_{t d}$ is the new occupancy state, $o s_{t d} \in\{$ Away, Sleeping, Active $\}$ (Away: when the occupant is not at home, Sleeping: when the occupant is at home but sleeping, and Active: when the occupant is at home and not sleeping). The agent first selects a new state as function of his occupancy pattern $o p_{d}$, previous state $o s_{(t-1) d}$, and time of day $t$, then decides the duration $d r$ of the state.
The PM distinguishes between tasks which can be performed by one occupant at a time and personal activities that can be performed by some/all occupants and can be shared. When the occupant is in the Active state, he/she can either select to start a task or personal activity, or decrement the duration of an ongoing activity. The action of selecting new activities is defined by the function $\mathrm{AC}:$ age, emp, $\mathbf{t}, \mathbf{d} \rightarrow\{\mathbf{0}, \mathbf{1}\}_{\mathbf{a c} / \mathbf{t k}}, \mathbf{d r}$ which is performed by the occupant agent for every personal activity $a c \in\{$ Using_the_computer, Watching_television, Listening_to_music, Taking_shower/bath $\}$ and task $t k \in$ $\{$ Preparing_food, Vacuum_cleaning, Ironing, Doing_dishes, Doing_laundry $\}$. The function returns a Boolean value $\{0,1\}$ to distinguish if the action will take place or not. This way of modelling enables the occupant to perform more than one activity at a time. The decision of doing an activity is based on the occupant age, employment type (emp), time of day $t$, and day type $d$. If a new activity is selected to be performed, the agent selects the duration $d r$ of the activity.

2) Occupant Location: Whenever the occupant agent is in the Active or Sleeping state, it means he/she should be in one of the house rooms. Every activity is assigned a set of possible rooms. The occupant agent determines his/her location $r_{t d}$ using the function $\mathbf{O L}: \mathbf{o s}_{\mathbf{t d}}, \mathbf{A} \mathbf{C}_{\mathbf{t d}}, \mathbf{T} \mathbf{K}_{\mathbf{t d}} \rightarrow \mathbf{r}_{\mathbf{t d}}$, where $A C_{t d}$ are ongoing personal activities, and $T K_{t d}$ are ongoing tasks. If the occupant is doing more than one activity at a time, he/she may have a set of possible rooms and his/her location alternates among these rooms at every time step.

3) Occupant Energy Awareness and Energy Usage: Occupants' energy awareness have been modelled in existing literature in different ways. For example, Carmenate et al. [9] distinguishes between energy literate and energy illiterate occupants. Similarly, Azar and Menassa [6] divided occupants into high, medium, and low consumers. Another way is using average yearly/daily consumption as a characteristic of the occupant [5]. The most detailed and flexible definition of energy awareness was proposed in Zhang et al. [7] where energy consumers can belong to one of four consumer types: 'Follower Green', 'Concerned Green', 'Regular Waster', and 'Disengaged Waster'. Based on the consumer type, the agent's energy awareness attribute is assigned a value between 0 and 100. This attribute is used to decide the probability that an occupant follows energy saving actions such as turning off devices when they are not in use. The value is calculated based on a normal distribution for every consumer type (Table I). In the current ABM, the energy awareness of occupant agents is defined based on occupant types in Zhang et al. [7].

TABLE I: Mean and Standard Deviation of Consumer Types

\begin{tabular}{lll}
\hline Consumer Types & Mean $\mu$ & Standard Deviation $\sigma$ \\
\hline Follower Green & 0.74 & 0.041 \\
\hline Concerned Green & 0.72 & 0.043 \\
\hline Regular Water & 0.41 & 0.033 \\
\hline Disengaged Waster & 0.25 & 0.057
\end{tabular}

The action of turning appliances on/off is defined by the function $\mathrm{TO}_{\mathbf{a}}: \mathbf{a c}_{\mathbf{t d}} \rightarrow \operatorname{turnOn_{\mathbf {a}}}$ $\mathbf{a c}_{\mathbf{t d}}, \mathrm{O}_{\mathbf{a}}$, ea $\rightarrow\{\text { keepOn, turnOff }\}_{\mathbf{a}}$. 
- Basic Model —_ Follower Greens _ Concerned Green —— Regular Waster — Disengaged Waster

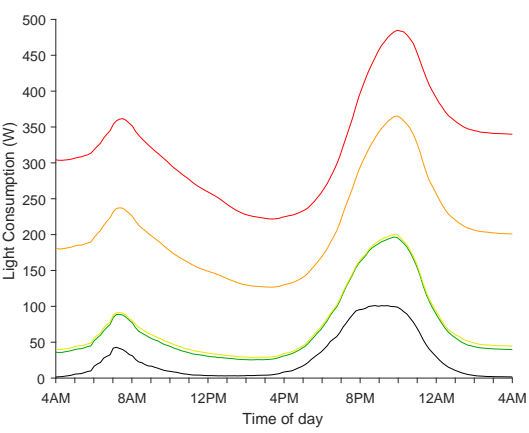

(a) Lights Consumption

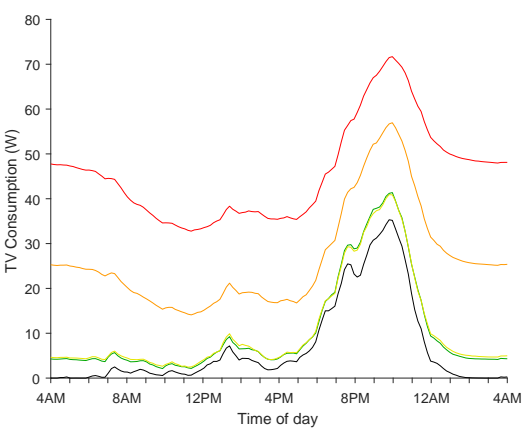

(b) TV Consumption

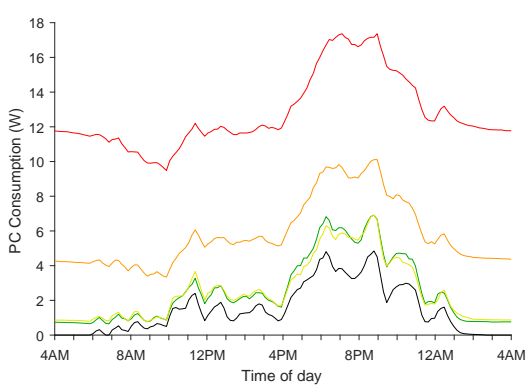

(c) PC Consumption

Fig. 1: Appliances energy consumption of one occupant (25-39 years old / full-time job)

When the occupant starts an activity $a c_{t d}$, he/she turns on the appliance $a$ associated to this activity. When the activity ends and based on the occupants energy awareness $e a$, he/she may turn off the appliance or keep it on. The occupant may also communicate with other occupant/s $O_{a}$ who may be using the same appliance at the same time to decide whether to turn off the appliance. The action of turning off appliances is also executed every time an occupant visits a room and finds appliances that are on but unused. The action of turning lights on/off is different from using appliances, because using lights depends on the amount of daylight and the location of occupants. $\mathbf{T O}_{\mathbf{r}}: \mathbf{r}_{\mathbf{t d}}$, daylight ${ }_{\mathbf{t d}} \rightarrow\{\text { turnOn, } \text { !turnOn }\}_{\mathbf{r}}$ $\mathbf{r}_{\text {td }}, \mathbf{O}_{\mathbf{r}}, \mathbf{e a} \rightarrow\{\text { turnOff, !turnOff }\}_{\mathbf{r}}$

Every time the occupant is in a location $r_{t d}$ he may decide to turn on the light in this room based on the amount of daylight at the timestep (daylight ${ }_{t d}$ ) [11]. When the occupant leaves his/her location, he/she checks if there is more occupants in the room $O_{r}$, and based on his energy awareness (ea) he/she may decide to turn off the light or not.

In summary, the occupant agent $\mathbf{O A}$ is defined using the set $<$ age, emp, $o p_{d}, o s_{t d}, e a, A C_{t d}, T K_{t d}, r_{t d}>$ and can perform the actions $<\mathbf{O S}, \mathbf{A C}, \mathbf{O L}, \mathbf{T O} \mathbf{O}_{\mathbf{a}}, \mathbf{T} \mathbf{O}_{\mathbf{r}}>$. The model was implemented in Repast Simphony (https://repast.github.io), a Java-based agent-based platform. The implementation of the occupancy and activity behaviour in ABM was tested and found to be generating the same occupant behaviour as the original PM [11]. Three appliances were implemented: Lights, TV, and PC, which are clearly affected by the energy awareness of occupants like leaving lights on when leaving a room or leaving the TV/PC on when the activity ends.

\section{Simulation Results and Discussion}

This section presents a set of experiments to test the validity of the model and study the effect of energy awareness on household consumption. In each scenario, the average energy consumption of 100 simulated households (with the same type and energy awareness, but different income, appliances number and types, and house rooms) is calculated.

\section{A. Experiment 1: Single Occupant Household}

The purpose of this experiment is to demonstrate the validity of the developed model. In order to study the effect of energy awareness, single occupant households were simulated varying ages and employment of occupants. Fig. 1 represents the resulting weekday average consumption of lights, TV, and PC for a 25-39 years old occupant in full-time job. Each of the sub-figures in Fig. 1 includes 5 scenarios: the basic model which is the ideal scenario with $100 \%$ energy awareness (referring to Aerts PM [11]), and four scenarios each with a different occupant type.

In the basic model, it is observed that when the occupant is sleeping or away the energy consumption is very low or almost zero. For the other four scenarios, it is observed that the implemented model produces very similar trend of daily energy consumption. The observed difference in energy consumption is due to the energy awareness attribute which has caused the line graph to level up in a proportionally based the energy awareness percentages in Table I. The energy consumption of Follower Green and Concerned Green occupants are almost similar because their mean energy awareness is very close (74\% and $72 \%$ respectively). While the two waster occupants are much higher with the Regular Waster being more efficient than the Disengaged Waster (41\% and 25\% respectively). Same observations were made for other day types, age groups, and employment types.

These results prove the validity of the implemented model that produces energy consumption trends similar to the basic model which was constructed from real data in the PM, and reflects the various energy awareness levels of occupants.

\section{B. Experiment 2: Two Occupant Household}

In order to study the effect of energy awareness on multiple occupant households, the energy awareness of occupants is reduced to the two extreme types: Follower Green (G) and Disengaged Waster $(\mathrm{W})$ which limits the number of scenarios while achieving the objectives of this study. The total energy consumption per day for the three appliances (lights, TV, and PC) was calculated and shown in Fig. 2 which shows the consumption of two 25-39 year old occupants (Fig. 2a where both are in full-time job, and Fig. $2 b$ where one is in full-time job and the other is unemployed). The legend of the figure encodes the energy awareness of the household, where the sequence of the letters ( $\mathrm{G}$ and $\mathrm{W}$ ) has the same sequence as 
the description of the household type in the captions of the sub-figures.

It is noticed that the observation in the previous experiment (one occupant household) still applies on two occupant households which proves that the model reflects energy awareness of occupants with multiple occupancy. Both Figures (2a and 2b) show the two extremes of energy consumption when there are two Follower Green occupants or two Disengaged Waster occupants at home. In-between scenarios in Fig. 2a resulted in the same energy consumption (yellow and orange crossed lines) even when reversing the energy awareness of the two occupants. However, this observation doesn't hold when having different employment types (Fig. 2b). It is observed that the household consumes less energy when the unemployed occupant is a Follower Green. This is observed during the whole 24 hours except few hours in the morning 7:00 am and 9:30 am) when it is more probable that the Disengaged Waster full-time occupant is awake and the Follower Green unemployed occupant is sleeping. Similar observation is noticed when the unemployed occupant is a Disengaged Waster where the household consumes more energy. This is explained by the fact that unemployed occupants spend more time in the house which makes their effect more obvious than full-time employed occupants.

These observations show that employment type is a factor that affects the energy consumption of the house when varying occupants energy awareness. However, further investigation is needed to quantify this effect and test it on other age groups and household types.

\section{COnClusion And Future Work}

This paper presented a methodology to integrate agentbased and probabilistic models to overcome limitations of existing models. The proposed hybrid model incorporates energy awareness at occupant level and produces fine-grained data to model behavioural energy waste. It was shown that the developed model produces valid consumption data compared to the real data and reflects various energy awareness levels of occupants. The experiments also showed that there is an effect for employment type on the energy consumption of the house. This conclusion needs to be quantified in future research, and other social parameters such as occupants ages and household types can also be studied to gain insights towards energy efficiency plans for families. Furthermore, the model opens the way for more experiments to study the effect of intervention technologies (e.g. customized energy waste messages) and family members pressure on the energy consumption of household.

\section{REFERENCES}

[1] O. T. Masoso and L. J. Grobler, "The dark side of occupants' behaviour on building energy use," Energy and Buildings, vol. 42, pp. 173-177, 2010.

[2] D. Aerts, J. Minnen, I. Glorieux, I. Wouters, and F. Descamps, "A method for the identification and modelling of realistic domestic occupancy sequences for building energy demand simulations and peer comparison," Building and Environment, vol. 75, pp. 67-78, 2014.

[3] J. Widén, M. Andreas, and K. Ellegårdc, "Models of domestic occupancy, activities and energy use based on time-use data : Deterministic and stochastic approaches," Journal of Building Performance Simulation, vol. 5, no. 1, pp. 27-44, 2012.

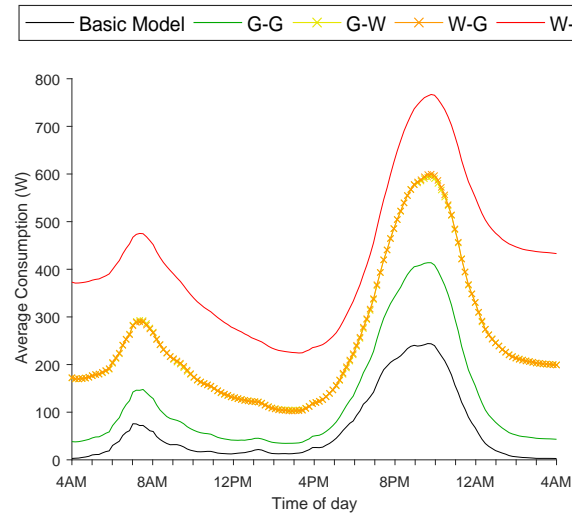

(a) Two occupants both in full-time job

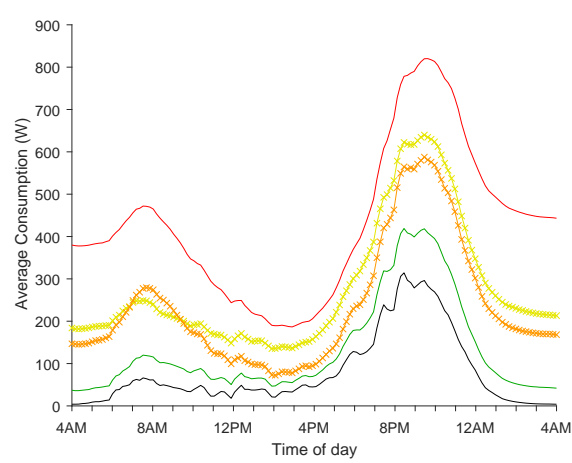

(b) Two occupants: the first in full-time job and the second is unemployed

Fig. 2: Total energy consumption of two occupant households both 25-39 years old

[4] I. Richardson, M. Thomson, D. Infield, and C. Clifford, "Domestic electricity use : A high-resolution energy demand model," Energy and Buildings, vol. 42, pp. 1878-1887, 2010.

[5] E. Azar and C. C. Menassa, "A comprehensive framework to quantify energy savings potential from improved operations of commercial building stocks," Energy Policy, vol. 67, pp. 459-472, 2014.

[6] _- "Agent-Based Modeling of Occupants and Their Impact on Energy Use in Commercial Buildings," Journal of Computing in Civil Engineering, vol. 26, no. 4, pp. 506-518, 2012.

[7] T. Zhang, P. O. Siebers, and U. Aickelin, "Simulating user learning in authoritative technology adoption: An agent based model for council-led smart meter deployment planning in the UK," Technological Forecasting and Social Change, vol. 106, pp. 74-84, 2016.

[8] E. Bonabeau, "Agent-based modeling: Methods and techniques for simulating human systems," Proceedings of the National Academy of Sciences, vol. 99, no. suppl 3, pp. 7280-7287, 2002.

[9] T. Carmenate, P. Inyim, N. Pachekar, G. Chauhan, L. Bobadillaa, M. Batoulib, and A. Mostafavi, "Modeling Occupant-Building-Appliance Interaction for Energy Waste Analysis," Procedia Engineering, vol. 145, pp. 42-49, 2016.

[10] U. Wilke, F. Haldi, and D. Robinson, "A bottom-up stochastic model to predict building occupants' time-dependent activities," Building and Environment, vol. 60, pp. 254-264, 2013.

[11] D. Aerts, "Simulations, Occupancy and Activity Modelling for Building Energy Demand, Comparative Feedback and Residential Electricity Demand Characteristics," PhD Thesis, Vrije University Brussel, 2015.

[12] Q. Reynaud, Y. Haradji, F. Sempé, and N. Sabouret, "Using TimeUse Surveys in Multi Agent Based Simulations of Human Activity," in Proceedings of the 9th International Conference on Agents and Artificial Intelligence - Volume 1: ICAART, Porto, Portugal, 2017, pp. 67-77.

[13] Eurostat, the statistical office of the European Union. (2003 onwards) Average number of rooms per-person and group by type of household income from 2003 - EU-SILC survey. (Accessed July 3, 2017). [Online]. Available: https://data.europa.eu/euodp/en/data/dataset/ pYzSXuZuS2yZzD3nQKQcWQ

[14] A. W. Evans and O. M. Hartwich, "Unaffordable Housing: Fables and Myths," Policy Exchange, Tech. Rep., 2005. 